\title{
The Optimization of Reactive Power for Distribution Network With PV Generation Based on NSGA-III
}

\author{
Yongle AI, Mingzhu DU, Zhihang PAN, and Gangxing LI
}

\begin{abstract}
A reactive power optimization algorithm for distribution network with photovoltaic (PV) generation is proposed to address the problems of power quality degradation, system active power losses increase and system instability caused by the connection of PV generation. Firstly, a multi-objective reactive power optimization model is established with the objective functions of minimizing system active power losses, controllable loads reduction, and PV active power reduction. Secondly, the PV power generation system is modeled mathematically. Thirdly, using the non-dominated sorting genetic algorithm (NSGA-III) to solve the model. Finally, the feasibility and validity of the proposed method are verified by the simulation with the modified IEEE123-bus system.
\end{abstract}

Index Terms-Distribution network, non-dominated sorting genetic algorithm (NSGA-III), photovoltaic power generation, reactive power optimization.

\section{INTRODUCTION}

$\mathrm{W}$ ITH the strong support of the National Energy Administration for subsidizing photovoltaic (PV) power generation projects, distributed PV power generation systems have been developed. However, after the PV is connected to the distribution system, the radiation structure will be changed, which will have a negative impact on the node voltage and tidal distribution, and also bring adverse effects on the stable system operation [1]. The study of reactive power optimization in distribution network with PV generation is of great importance to reduce system active power losses and ensure the safe and stable operation of distribution networks [2].

Initially, most of the optimization of reactive power only focus on single-objective, such as minimizing system cost [3], system active power losses [4], or emissions [5]. With the improvement of power system operation level, reactive power optimization is focusing on a multi-objective optimization that integrates operation indexes, economic indexes, and power quality indexes, etc. The traditional methods of solving multi-objective problems are to convert multi-objective optimi-

Manuscript received April 20, 2021; revised June 13, 2021; accepted August 10, 2021. Date of publication September 30, 2021; date of current version September 22, 2021. This work was supported in part by the Key Research Projects of Higher Education Institutions of Henan Provincial Education Department under Grant 17A470001 and in part by Henan Province Science and Technology Research Project under Grant 172102310569.

All authors are with the School of Electrical Engineering and Automation, Henan Polytechnic University, Jiaozuo, 454150, China (e-mail: 1445524547@qq.com).

Digital Object Identifier 10.24295/CPSSTPEA.2021.00017 zation into single-objective by weighting methods [6], fuzzy decision methods [7], and $\varepsilon$ constraint methods, and so on. However, there are many limitations in these methods, and the solution results are often unsatisfactory. Multi-Objective Evolutionary Algorithm (MOEA) provides another way to solve these problems, and common algorithms used in this field are Multi-Objective Differential Evolution (MODE) [8], Non-Dominated Sorting Genetic Algorithm (NSGA) [9], Strength Pareto Evolutionary Algorithm (SPEA) [10], Multi-Objective Genetic Algorithm (MOGA) [12], and so on.

The common optimization models are those with two or three objectives. In [8], the objective model for minimizing fuel cost and active power losses was developed and solved using MODE. The literatures [9], [10] consider both system active power losses and voltage quality and solves them using NSGA-II and SPEA-II, respectively. The literature [11] takes minimizing generation cost, system active power losses, and controllable load (CL) cost as objectives. The literature [12] used MOGA to minimize system active power losses, voltage deviation, and voltage stability coefficient. The literatures [13], [14] aim to minimize generation cost, system active power losses, and voltage stability enhancement factor, which are solved using cluster-based adaptive teaching and particle swarm algorithms, respectively.

NSGA is one of the most representative algorithms for solving high-dimensional multi-objective optimization problems. Nowadays, NSGA-III uses a reference point-based selection operation instead of a congestion distance selection operation, which makes up for the shortcomings of NSGA-II and effectively improves the population diversity. In this paper, NSGA-III is proposed to solve the optimization of reactive power for distribution network with PV generation by minimizing system active power losses, controllable loads (CLs) reduction, and PVs active power reduction. NSGA-III provides a novel idea for the optimization of reactive power for distribution network with PV generation.

The remainder of this paper is organized as follows: Section II describes the multi-objective reactive power optimization model of distribution network with PVs. Section III presents the PV system modeling. Solving the problem using NSGA-III and reactive power optimization flow is described in Section IV. Section V presents simulation results and discussion. Finally, the conclusions of this paper are summarized in the last part. 
II. Multi-Objective Reactive Power Optimization Model of DistRibution Network With PVS

\section{A. Objective Functions}

Considering the stability and economy of distribution network operation, this paper establishes the objective functions of minimizing system active power losses, CLs reduction, and PVs active power reduction.

1) Objective Function $f_{1}$ : Minimize System Active Power Losses $P_{L}$

The system active power losses are one of the major indicators reflecting the operation level of the distribution network [15], which mainly refer to the active power losses on the lines.

$$
\min f_{1}=\min P_{\mathrm{L}}=\sum_{l=1}^{n_{2}} R_{l} \frac{P_{l}^{2}+Q_{l}^{2}}{U_{l}^{2}}
$$

Where $l$ and $n_{2}$ denote the branches number and the total branches number, respectively; $R_{l}$ is the resistance on branch $l$. $U_{l}, P_{l}$ and $Q_{l}$ denote the voltage, active power as well as reactive power at the end of branch $l$, respectively.

\section{2) Objective Function $f_{2}$ : Minimize the Percentage Reduction of CLs}

There are a lot of CLs that can be reduced and have relatively low requirements for power supply reliability in the distribution network, such as air conditioning loads and refrigerator loads. The reduction of CLs can be achieved by controlling the start/ stop time of air conditioners or refrigerators and adjusting the set temperature. Usually, the loads power reduction cannot exceed the upper limit of the loads reduction potential.

$$
\min f_{2}=\min \mathrm{CL}(\%)=100\left(1-\frac{\sum_{\text {all CLs }} \ell_{i} P_{\mathrm{L}, i}}{\sum_{\text {all CLs }} P_{\mathrm{L}, i}}\right)
$$

Where $P_{\mathrm{L}, i}$ is the loads power without reduction; parameter $\ell_{i}$ denotes the CLs reduction factor, $\ell_{i, \text { min }}<\ell_{i} \leqslant 1$, and $\ell_{i \text {, min }}$ is taken as 0.9 in this paper.

\section{3) Objective Function $f_{3}$ : Minimize the Percentage Reduction of PVs Active Power}

The PV inverter can reduce the active power output of PVs to achieve the purpose of voltage qualification and minimum the reduction of PVs active power.

$$
\min f_{3}=\min \mathrm{PV}(\%)=100\left(1-\frac{\sum_{\text {all PVs }} T_{i} I_{\mathrm{rr}, i} P_{\mathrm{PV}, i}}{\sum_{\text {all PVs }} I_{\mathrm{rr}, i} P_{\mathrm{PV}, i}}\right)
$$

Where $P_{\mathrm{PV}, i}$ represents the rated power; $I_{\mathrm{rr}, i}$ is the irradiance; $T_{i}$ is the PVs reduction factor, which is taken from the set $\{0$, $0.1,0.2,0.3, \ldots, 0.9,1\}$.

\section{B. Constraints}

The constraints for reactive power optimization include equation constraints and inequality constraints. The equation constraints mainly refer to the tide constraint [16], which can be expressed as (4).

$$
\left\{\begin{array}{l}
P_{\mathrm{PV}, i}-P_{\mathrm{L}, i}-U_{i} \sum_{i=1}^{N} U_{j}\left(G_{i j} \cos \delta_{i j}+B_{i j} \sin \delta_{i j}\right)=0 \\
Q_{\mathrm{PV}, i}+Q_{C, i}-Q_{\mathrm{L}, i}-U_{i} \sum_{i=1}^{N} U_{j}\left(G_{i, j} \sin \delta_{i, j}-B_{i, j} \cos \delta_{i, j}\right)=0
\end{array}\right.
$$

Where $N$ denotes the total nodes number; $G_{i j}$ denotes the conductance on line $i j$, and $B_{i j}$ denotes the susceptance; $P_{\mathrm{PV}, i}$ and $P_{\mathrm{L}, i}$ denote the PVs input active power and loads absorb active power at the $i_{\mathrm{th}}$ node respectively, and similarly $Q_{\mathrm{PV}, i}$ and $Q_{C, i}$ denote the PVs output reactive power and capacitors supply reactive power at the $i_{\mathrm{th}}$ node respectively.

Inequality constraints are a series of security constraints, including control variable constraints and state variable constraints [17]. In this paper, the control variables include PVs output power, voltage regulators (VRs) taps position, switching capacitors (SCs) state, and CLs reduction. The state variables include node voltage amplitude, line current, and electrical equipment capacity, which are shown through (5)-(12).

$$
\begin{gathered}
P_{\mathrm{PV}, \text { min }} \leqslant P_{\mathrm{PV}} \leqslant P_{\mathrm{PV}, \text { max }} \\
Q_{\mathrm{PV}, \text { min }} \leqslant Q_{\mathrm{PV}} \leqslant Q_{\mathrm{PV}, \text { max }} \\
T_{\mathrm{p}, \text { min }} \leqslant T_{\mathrm{p}} \leqslant T_{\mathrm{p}, \text { max }} \\
N_{\text {min }} \leqslant N \leqslant N_{\text {max }} \\
P_{\mathrm{L}, \text { min }} \leqslant P_{\mathrm{L}} \leqslant P_{\mathrm{L}, \text { max }} \\
0.95 \leqslant V_{i} \leqslant 1.05 \\
0 \leqslant I_{i} \leqslant I_{i, \text { max }} \\
C_{i, \text { min }} \leqslant \mathrm{C}_{i} \leqslant C_{i, \text { max }}
\end{gathered}
$$

Where $P_{\mathrm{PV}}, Q_{\mathrm{PV}}, T_{\mathrm{p}}, N, P_{\mathrm{L}}, V_{i}, I_{i}, C_{i}$ denote PVs active power, PVs reactive power, VRs taps position, SCs state change, CLs reduction power, node voltage, line current and electrical equipment (such as transformer, induction motor or generator, etc.) capacity, respectively, and max and min are their upper bounds and lower bounds.

\section{PV System Modeling}

The PVs power generation system includes the PVs panels, a first-stage DC/DC link and a second-stage DC/AC link [18]. The first-stage DC/DC link can realize the boost function and achieve the maximum power point tracking (MPPT) control, while the second-stage DC/AC link mainly performs the inverter control to achieve the grid-connected role. The block diagram of the grid-connected PV system is shown in Fig. 1.

The power injected into the grid by PVs is calculated as follows:

$P_{\mathrm{PV}}=m V_{\mathrm{PV}} V_{\mathrm{PCC}}\left[G \cos \left(\theta_{\mathrm{PV}}-\theta_{\mathrm{PCC}}\right)+B \sin \left(\theta_{\mathrm{PV}}-\theta_{\mathrm{PCC}}\right)\right]$ 


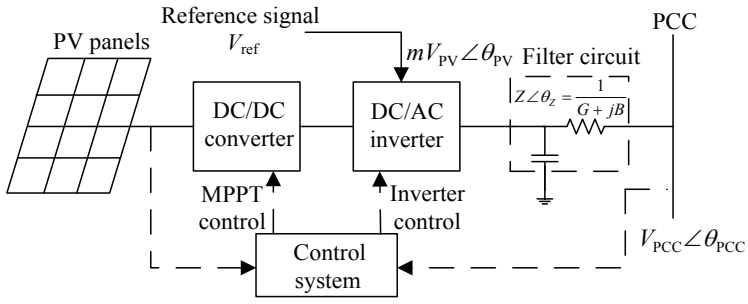

Fig. 1. Block diagram of the grid-connected PV system.

$$
Q_{\mathrm{PV}}=m V_{\mathrm{PV}} V_{\mathrm{PCC}}\left[G \sin \left(\theta_{\mathrm{PV}}-\theta_{\mathrm{PCC}}\right)-B \cos \left(\theta_{\mathrm{PV}}-\theta_{\mathrm{PCC}}\right)\right](14)
$$

Where, $m$ is the inverter modulation ratio and usually taken between 0 and 1 , which can regulate the PVs output voltage. $V_{\mathrm{PV}}$ and $V_{\mathrm{PCC}}$ are $\mathrm{PVs}$ output voltage and grid voltage. $\theta_{\mathrm{PV}}{ }^{-}$ $\theta_{\mathrm{PCC}}$ is their phase difference. $G$ is the line equivalent conductance. $B$ is the line equivalent susceptance.

In addition, the power injected into the grid by PVs is also limited by many factors, such as grid voltage, inverter power ratings, and harmonics [19]. The power injected into the PCC by PVs under grid voltage limitation is given by $S=P+j Q=U I^{*}$ to obtain (15).

$$
\begin{aligned}
P_{\mathrm{PCC}}+j Q_{\mathrm{PCC}}= & \left(V_{\mathrm{PCC}} \angle \theta_{\mathrm{PCC}}\right) . \\
& \left\{\frac{\left[\left(m V_{\mathrm{PV}} \angle \theta_{\mathrm{PV}}\right)-\left(V_{\mathrm{PCC}} \angle \theta_{\mathrm{PCC}}\right)\right]}{Z \angle \theta_{Z}}\right\}
\end{aligned}
$$

Where $Z \angle \theta_{Z}=1 /(G+j B)$ is the equivalent impedance of the line. The above equation is simplified to obtain (16).

$$
\begin{aligned}
P_{\mathrm{PCC}}+j Q_{\mathrm{PCC}}= & \frac{V_{\mathrm{PCC}} m V_{\mathrm{PV}}}{Z} . \\
& \angle\left(\theta_{\mathrm{PCC}}+\theta_{Z}+\theta_{\mathrm{PV}}\right)-\frac{V_{\mathrm{PCC}}^{2}}{Z} \angle \theta_{Z}
\end{aligned}
$$

Using Euler's formula,

$$
\begin{gathered}
{\left[P_{\mathrm{PCC}}+\frac{V_{\mathrm{PCC}}^{2} \cos \left(\theta_{Z}\right)}{Z}\right]+j\left[Q_{\mathrm{PCC}}+\frac{V_{\mathrm{PCC}}^{2} \sin \left(\theta_{Z}\right)}{Z}\right]=} \\
{\left[\left(\frac{V_{\mathrm{PCC}} m V_{\mathrm{PV}}}{Z}\right) e^{j\left(\theta_{\mathrm{PCC}}+\theta_{\mathrm{Z}}-\theta_{\mathrm{PV}}\right)}\right]}
\end{gathered}
$$

Assuming that $V_{\mathrm{PCC}}$ and $Z$ in (17) are constant values, $0 \leqslant$ $m \leqslant 1$, and $0 \leqslant \theta_{\mathrm{PV}}-\theta_{\mathrm{PCC}} \leqslant 2 \pi$, PVs injected $\mathrm{PCC}$ power diagram in the $\mathrm{PQ}$ plane under grid voltage limitation is shown in Fig. 2, which the center of the circle is $-\left[V_{\mathrm{PCC}}^{2}(G-j B)\right]$ and the radius is $\left[\left(m V_{\mathrm{PV}} V_{\mathrm{PCC}}\right) / Z\right]$. The circle radius changes with $m$ when $\left(\theta_{\mathrm{PV}}-\theta_{\mathrm{PCC}}\right)$ is constant. The operating point on the circle can be traced according to the change of $\left(\theta_{\mathrm{PV}}-\theta_{\mathrm{PCC}}\right)$ when $m$ is constant. For example, when $m=1$ and $\theta_{\mathrm{PV}}-\theta_{\mathrm{PCC}}=0$, it can be seen from Fig. 2 that PVs operate in the first quadrant that both active and reactive power is positive.

Under grid voltage and inverter rated power limitation, the operable overlap zone of PVs is shown in Fig. 3. In addition, the PVs operable zone is restricted to the dark half-circle part

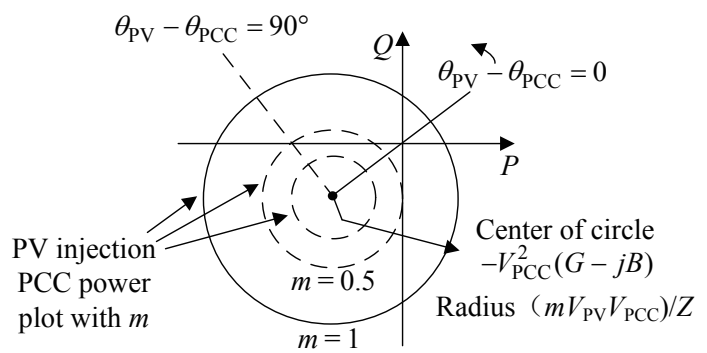

Fig. 2. Power diagram under PV injected PCC with grid voltage limitation on PQ plane.

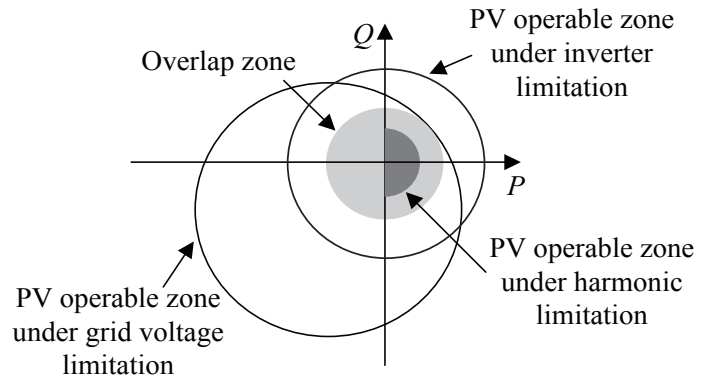

Fig. 3. PV operable zone on the $P Q$ plane.

of Fig. 3 in order to meet the grid harmonic criteria [19].

Therefore, the PVs system model in this paper is established as follows:

$$
\begin{gathered}
P_{\mathrm{PV}}=I_{\mathrm{rr}} P_{\mathrm{PV}}^{r} \\
Q_{\mathrm{PV}}=\left\{\begin{array}{l}
\sqrt{\left(P_{\mathrm{PV}}^{r}\right)^{2}-P_{\mathrm{PV}}^{2}}, P_{\mathrm{PV}}>0.1 P_{\mathrm{PV}}^{r} \\
0, P_{\mathrm{PV}} \leqslant 0.1 P_{\mathrm{PV}}^{r}
\end{array}\right.
\end{gathered}
$$

Where $I_{\mathrm{rr}}$ represents the standardized value of irradiance and takes value in the range of 0 to 1 , and $P_{\mathrm{PV}}^{\mathrm{r}}$ refers to the rated power. From (19), it is shown that the PVs active power is bounded by $10 \%$ of the rated value and when exceeding the bound, $Q_{\mathrm{PV}}$ is positive and will provide reactive power, otherwise, $Q_{\mathrm{pV}}$ is equal to 0 . So the active and reactive power of PVs should be positive. When $\theta_{\mathrm{PV}}-\theta_{\mathrm{PCC}}=0$, PVs can provide extra power. In this paper, we focus on the optimal response of the $\mathrm{PV}$-containing distribution network and therefore ignore the $10 \%$ threshold limit for PVs supply.

\section{NSGA-III-Based Reactive Power Optimization}

\section{A. Overview of NSGA-III}

Firstly, an initial population set $P$ with size $N$ is given. Then, offspring population set $Q$ with size $N$ is generated by crossover and mutation operations. It needs to select $N$ better individuals from $R(R=P \cup Q)$ through the non-dominated sorting mechanism to form a new generation population. Then, divide $R$ into different non-dominant layers $F_{1}, F_{2}, \ldots, F_{n}$, and then the 


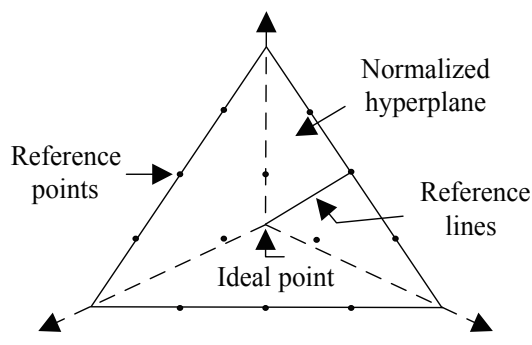

Fig. 4. Hyperplane reference point distribution.

non-dominated set with high priority is retained in the next generation.

When $\left|F_{1} \cup F_{2} \cup \cdots F_{l-1}\right|<N$ and $\left|F_{1} \cup F_{2} \cup \cdots F_{l}\right|>N$, define $F_{1}$ as the critical layer, and select $N$ individuals into the offspring based on the critical layer.

\section{B. Non-Dominated Sorting}

Firstly, it should define $x_{i}$ as the solution and $F_{j}$ as the set. If $x_{i}$ belongs to $F_{j}, x_{i}$ can be written as $x_{i j}$.

Then, $n_{i}$ and $S_{i}$ are defined, which denote the number of solutions $x_{i}$ that dominates individual $i$ and the set of solutions $x_{i}$ that is dominated by individual $i$, respectively. These two parameters $n_{i}$ and $S_{i}$ can be compared with all other solutions in the population except $x_{i}$ itself. Once $x_{i}$ belongs to the set $F_{j}, n_{i}$ and $S_{i}$ can be written as $n_{i j}$ and $S_{i j}$.

The first step requires determining $n$ and $S$ in the population, and find all solutions with $n=0$, and saving them to the first set $F_{1}$.

The second step needs to find all solutions in the set $F_{1}$. It should examine the individual set $S_{i 1}$ dominated by each solution $x_{i 1}$, and if $x_{q}$ belongs to $S_{i 1}$, then $n_{q}-1, n_{q \text {, new }}=n_{q \text {, old }}-1$. When $n_{q \text {, new }}=0$, deposits $x_{q}$ into the next set $F_{2}$, and so on.

\section{Determination of Hyperplane Reference Points}

NSGA-III uses the boundary-crossing construction weighting method for reference point selection [20]. In the normalized hyperplane, it is assumed that each dimensional target is divided into $p$ parts which are equal, at which point the number of reference points $H$ is calculated as follows:

$$
H=\left(\begin{array}{l}
M+p-1 \\
p
\end{array}\right)
$$

Such as in a 3-dimensional optimization problem, each dimensional objective is quadratically divided, results in 15 reference points, which are uniformly distributed as shown in Fig. 4. When the reference points are broadly and evenly distributed on the normalized hyperplane, the selected population will be also broadly and evenly distributed on the true Pareto surface.

\section{Adaptive Normalization of Populations}

The minimum value of $z_{i}^{\min }(i=1,2, \ldots, M)$ for each dimensional objective of the individuals in the current population $S_{t}$ is selected to constitute the ideal point of the current population $\bar{z}=\left(z_{1}^{\min }, z_{2}^{\min }, \cdots, z_{M}^{\min }\right)$. Shift the population $S_{t}$ according to

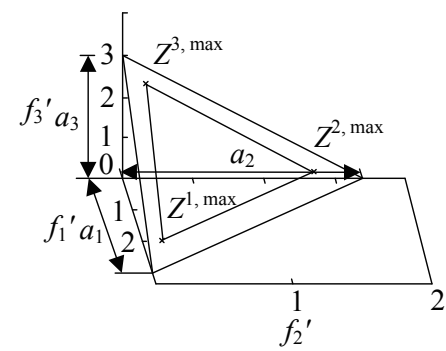

Fig. 5. Hyperplane in 3-dimensional target.

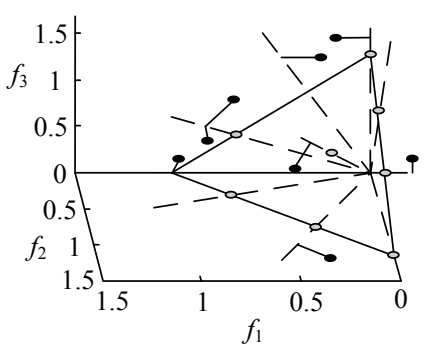

Fig. 6. Individual and reference point association operations.

$f_{i}^{\prime}(x)=f_{i}(x)-z_{i}^{\min }$, so that the ideal point becomes the origin.

Then, the extreme value $z^{i, \max }$ of each dimensional coordinate takes the minimum of the scalar function of (21).

$$
\operatorname{ASF}(x, \omega)=\max _{i=1}^{M} f_{i}^{\prime}(x) / \omega_{i}, x \in S_{t}
$$

Where, $\omega$ is the unit direction vector of the coordinate axis, and the $M$-dimensional linear hyperplane is constructed using $M$ polar points through (22) with an intercept of $a_{i}$ in each dimension, as shown in Fig. 5.

$$
f_{i}^{n}(x)=\frac{f_{i}^{\prime}(x)}{a_{i}-z_{i}^{\min }}=\frac{f_{i}(x)-z_{i}^{\min }}{a_{i}-z_{i}^{\min }}(i=1,2, \cdots, M)
$$

Where, $\sum_{i=1}^{M} f_{i}^{n}=1$

\section{E. Association Operation}

The association operation allows the individuals in the population to be associated with the corresponding reference points respectively. Firstly, the line between the origin and the reference point is used as the reference line of the reference point in the target space, as shown by the dashed line in Fig. 6 . Then, the distances of the individuals in $S_{t}$ to each reference line are calculated, and the individuals are associated with the corresponding reference points when they are closest to the reference line. Fig. 6 gives an example of a 3-dimensional target, where the gray points represent reference points and the black points represent individuals in the target space, and the individual finds the reference line at the closest distance to it, respectively, and then associates it with the corresponding reference point. 


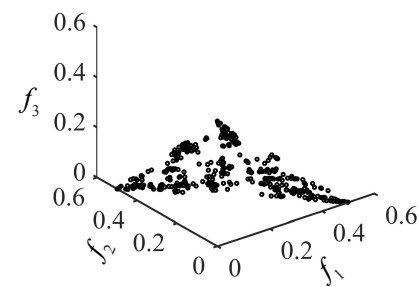

(a) NSGA-II

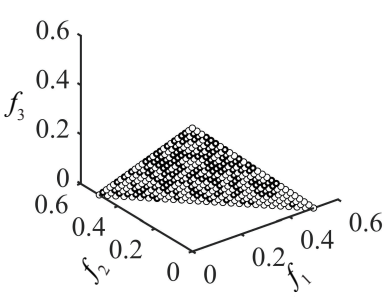

(b) NSGA-III
Fig. 7. Solution sets of two algorithms on DTLZ1.

\section{F. Individual Retention Operation}

After the association operation, the following situations may occur. First, the reference point is associated with one or more individuals. Second, no individual is associated with it. NSGA-III records the number of individuals $\rho_{j}$ associated with reference point $j$ in the set $P_{t+1}=S_{t} / F_{l}$.

Firstly, it needs to select the reference point $J_{\min }$ with the lowest number of associations, and if there is more than one such reference point, $j$ is randomly selected from it.

If $\rho_{j}=0$, it means that there is no individual associated with it in $P_{t+1}$. At this point, two cases are possible. First, if there is/are one or more individuals associated with it in $F_{l}$, then the individual nearest to the reference line corresponding to reference point $j$ is associated $\left(\rho_{j}=\rho_{j}+1\right)$ and the individual is added to $P_{t+1}$. Second, if there is no individual associated with reference point $j$ in $F_{l}$, then the reference point is not considered in the rest of the operation.

When $\rho_{j} \geqslant 1$, it means that there is/are one or more individuals associated with it in $P_{t+1}$. Then a reference point $j$ is randomly selected, and if there is an individual in $F_{l}$ associated with reference point $j\left(\rho_{j}=\rho_{j}+1\right)$, then that individual is added to $P_{t+1}$.

Repeat the above operations until the size of $P_{t+1}$ is equal to $N$.

\section{G. Algorithm Test}

In this paper, NSGA-III is tested by using the 3-dimensional objective function DTLZ1 [21]. In order to take full account of the diversity of the population without adding computational complexity, the population size is set to 400 , and the number of iterations is set to 500 in the test experiment. The test results are shown in Fig. 7. Obviously, NSGA-III has a more uniform Pareto front distribution than NSGA-II, which can better solve the high-dimensional multi-objective optimization problem.

\section{H. Flow Chart of Reactive Power Optimization}

The flow chart for solving the proposed problem of reactive power optimization by using NSGA-III is shown in Fig. 8.

\section{Simulation Results and Discussion}

The feasibility of NSGA-III is verified by testing on the IEEE123-bus system with a voltage level of $4.16 \mathrm{kV}$ [22]. The test system is slightly modified, and the structure diagram of the modified IEEE123-bus system is shown in Fig. 9.

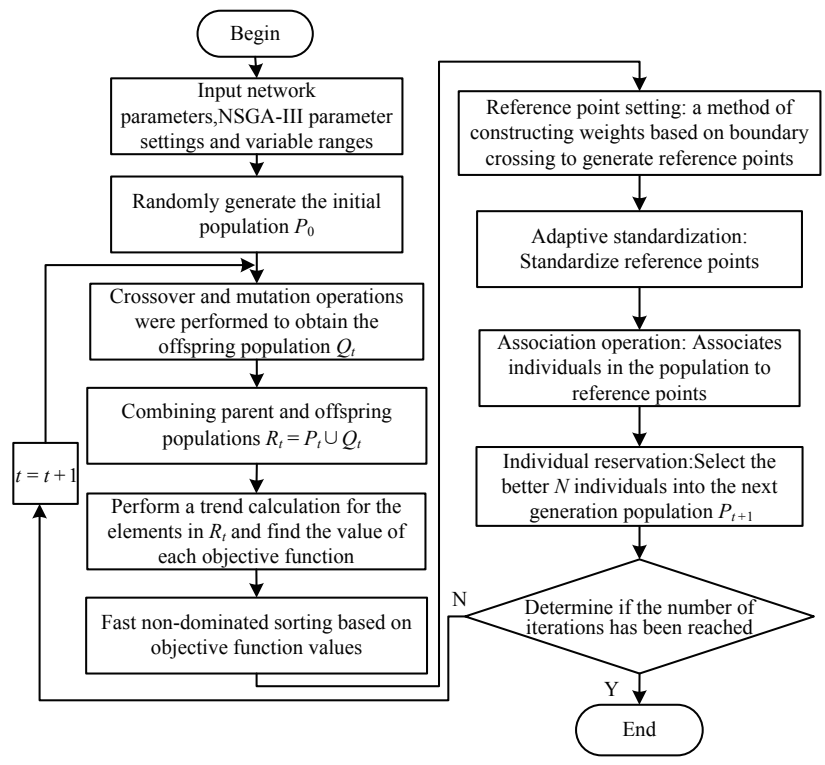

Fig. 8. Flow chart of reactive power optimization solution.

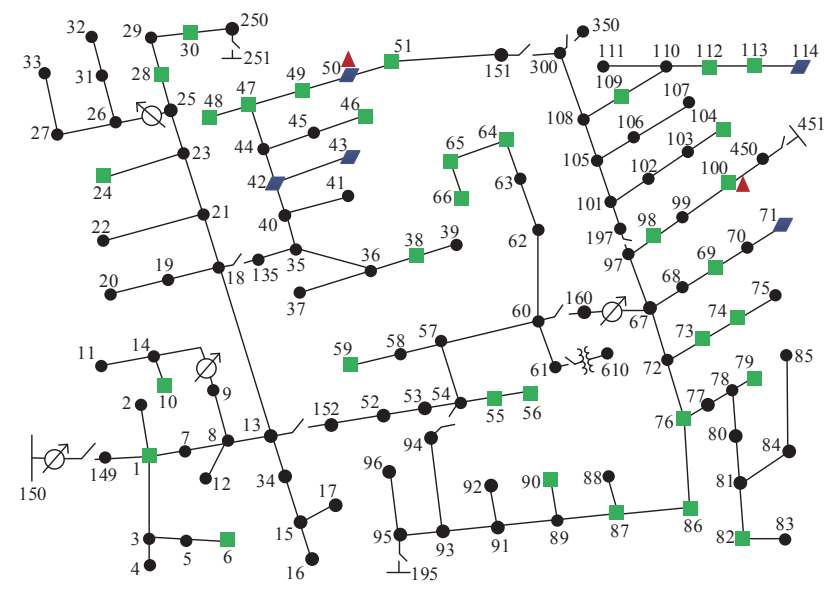

Fig. 9. Structure diagram of modified IEEE123-bus distribution system.

In which, the triangles indicate the installation locations of SCs, and nodes 50 and 100 are configured with 20 groups of three-phase switching capacitors, each group of SCs has a capacity of $10 \mathrm{kVar}$. The parallelograms indicate the installation locations of CLs, and the $A$-phase of nodes 71, 42, and 114, the $B$-phase of node 43 , and the $C$-phase load of node 50 are selected as CLs with reduction of no more than $10 \%$. The squares indicate the installation positions of PVs, as shown in Fig. 9, respectively. In addition, the rated output power of PVs is set to $1 \mathrm{MW}$. The variable ratio of VRs is $0.95-1.05$. The upper and lower steps are \pm 16 , and the step amount is $0.625 \%$. The illustration about the installation positions of PVs is shown in Fig. 10.

In the base case, SCs are not connected, VRs are in nominal tap position (0\%), PVs operate at unity power factor, and CLs are not reduced. Then the backward/forward sweep method is used for power flow calculation, and the results are simulated in the MATLAB environment [23]. The simulation modeling of power system components is based on the [23]. The normalized irradiation data and load data in one day $(0: 00-24: 00)$ 


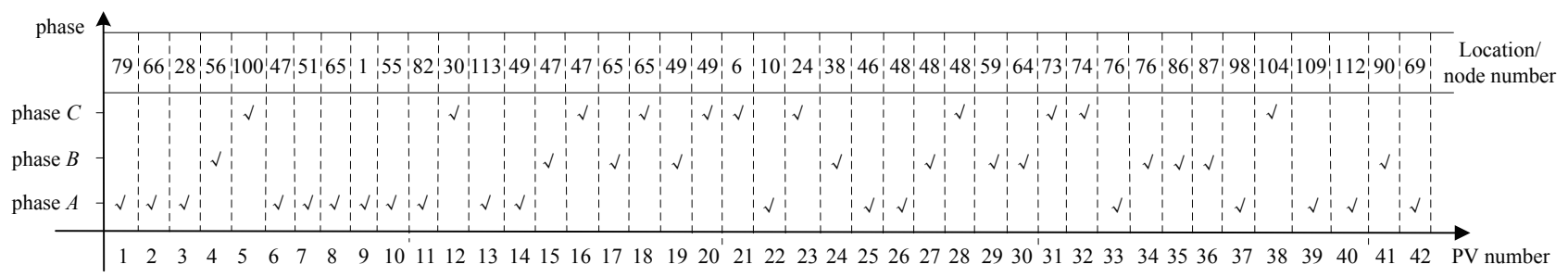

Fig. 10. Distribution diagram of PVs installation location.

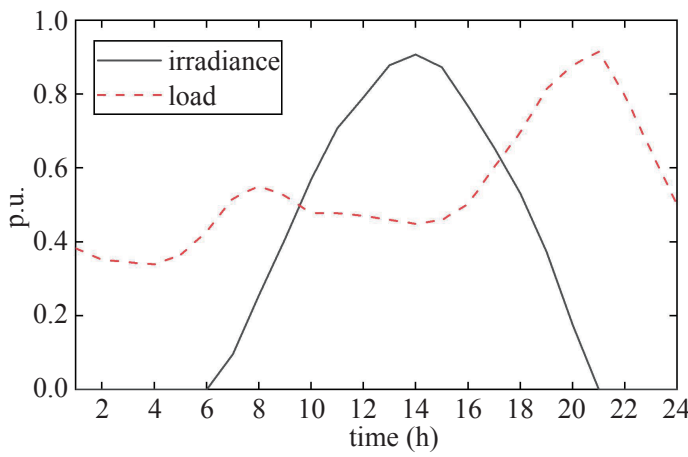

(a) Irradiation and load curve

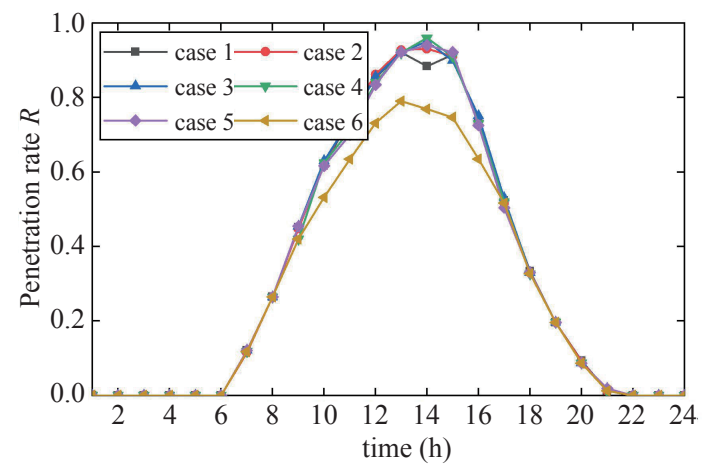

(b) Permeability rate $R$

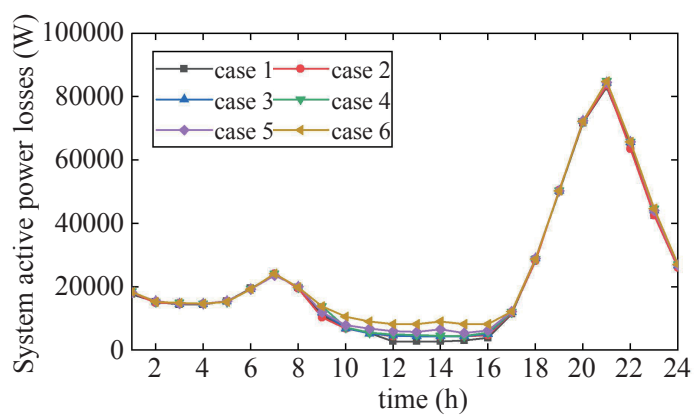

(c) System active power losses

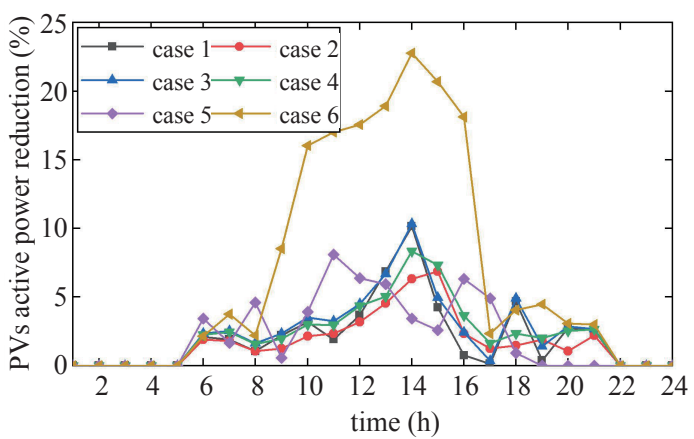

(d) PVs active power reduction

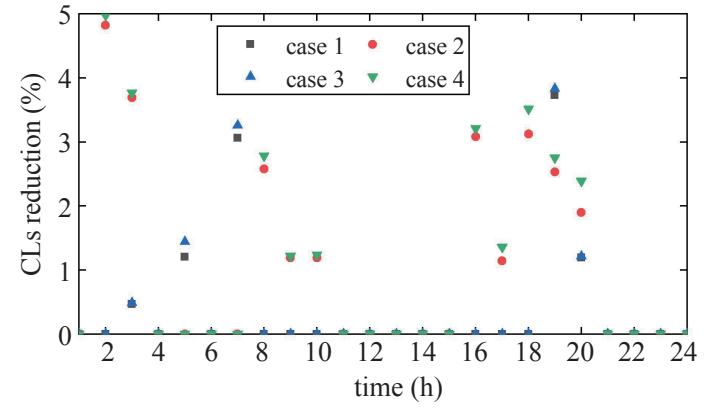

(e) CLs reduction

Fig. 11. Simulation results of IEEE123 system.

are shown in Fig. 11(a).

Six cases are simulated by solving the problem with NSGA-III, NSGA-II, and MOGA, respectively. Case 1: optimized with NSGA-III and PVs provide reactive power. Case 2: optimized with NSGA-III and PVs do not provide reactive power. Case 3: optimized with NSGA-II and PVs provide reactive power. Case 4: optimized with NSGA-II and PVs do not provide reactive power. Case 5: optimized with MOGA and PVs provide reactive power. Case 6: optimized with MOGA and PVs do not provide reactive power.

The performance of the optimization algorithm is evaluated using penetration rate $R$, as shown in Fig. 11(b). System active power losses are shown in Fig. 11(c). Comparing these two graphs, it can be seen that system active power losses are significantly lower when $R$ is higher. Permeability rate $R$ and system active power losses during peak hours of $10: 00$ AM to 5:00 PM are shown in Fig. 12(a) and (b), respectively. The least system active power losses are developed by using NSGA-III compared with NSGA-II and MOGA.

Total system active power losses during peak hours are shown in Table I. It can be seen that when PVs provide reactive power, system active power losses generated by NSGA-III are reduced by $2.97 \%$, NSGA-II by $3.91 \%$, and MOGA by $28.66 \%$. Therefore, it helps reduce system active power losses when PVs provide reactive power.

As shown in Fig. 11(e), MOGA has zero CLs reduction regardless of whether PVs have reactive support. It can be obtained that NSGA-III has a smaller reduction than NSGA-II. Comparing case 1 with case 2, case 1 has better performance. Similarly, case 3 has better results than case 4 . Therefore, it helps to decrease the reduction of CLs when PVs provide reactive power.

The reduction of PVs active power, as shown in Fig. 11(d). Except for case 6 where active power reduction can reach 


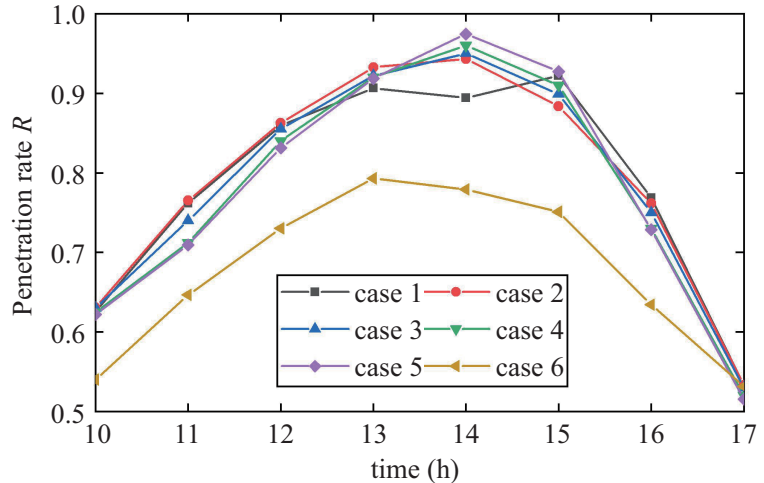

(a) Permeability rate $R$ during peak hours

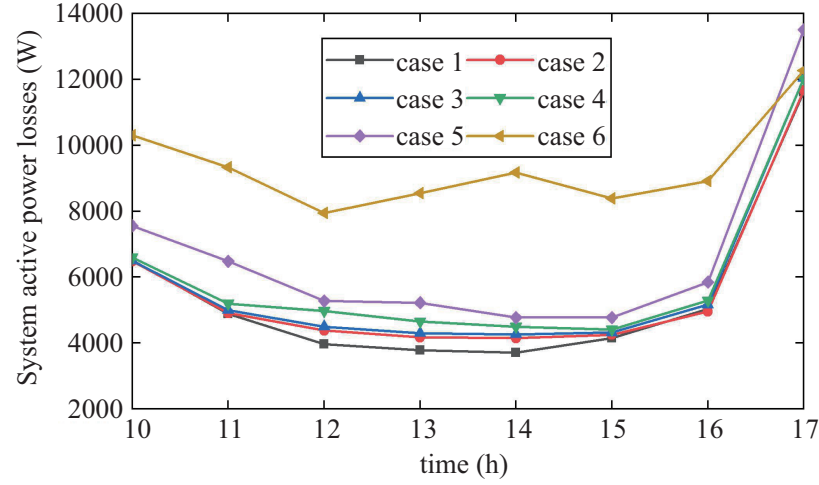

(b) System active power losses during peak hours

Fig. 12. Partial expend diagram of permeability rate $R$ and system active power losses.

TABLE I

Total System Active Power Losses Data Table During Peak Hours

\begin{tabular}{lccc}
\hline \hline & \multicolumn{2}{c}{ System active power losses/kW } & Improvement percentage/\% \\
\cline { 2 - 3 } & PVs provide reactive power & PVs don’t provide reactive power & 2.97 \\
NSGA-III & 43.639 & 44.973 & 3.91 \\
NSGA-II & 47.160 & 49.078 & 28.66 \\
MOGA & 53.436 & 74.889 & 28 \\
\hline \hline
\end{tabular}

about $25 \%$, only less than $10 \%$ of PVs active power is reduced in all other cases. The reduction of PVs active power is relatively lowest when using NSGA-III.

Obviously, NSGA-III with PVs reactive power support achieves more satisfactory effect for each objective function in this paper, which means that NSGA-III has better performance in reactive power optimization.

\section{CONCLUSION}

In this paper, a multi-objective reactive power optimization model, which includes minimizing system active power losses, PVs reduction, and CLs reduction, is constructed and solved by NSGA-III. The simulation is verified in the modified IEEE123bus distribution system. The main conclusions of this paper can be concluded in the two aspects.

1. NSGA-III has a more uniform Pareto distribution, which can better solve the multi-objective reactive power optimization problems.

2. It is beneficial to system operation and helps reduce system active power losses when PVs provide reactive power.

\section{REFERENCES}

[1] F. Gao, D. Qi, and G. Zou, "Research on voltage control of distribution network based on constraint programming," in Proceedings of 2020 Chinese Control and Decision Conference (CCDC), Hefei, China, 2020, pp. $1328-1333$.

[2] J. Wu, C. Shi, M. Shao, and R. Cai, "Reactive power optimization of a distribution system based on scene matching and deep belief network," in Energies, vol. 12, no. 17, p. 3246, Aug. 2019.

[3] A. Ali, D. Raisz, and K. Mahmoud, "Optimal capacity of PV inverter considering reactive power capability and active power curtailment," in Proceedings of 2017 Nineteenth International Middle East Power Systems Conference (MEPCON), Cairo, Egypt, 2017, pp. 1411-1415.
[4] H. Liu, G. Huang, C. Wang, H. Liu, Z. Wang, Z. Xu, and L. Shi, "Reactive power optimization of power grid with photovoltaic generation based on improved particle swarm optimization," in Proceedings of 2019 IEEE Innovative Smart Grid Technologies Asia (ISGT Asia), Chengdu, China, 2019, pp. $1536-1540$.

[5] S. S. Reddy and J. A. Momoh, "Minimum emissions optimal power flow in wind-thermal power system using Opposition based Bacterial Dynamics algorithm," in Proceedings of 2016 IEEE Power and Energy Society General Meeting (PESGM), Boston, MA, USA, 2016, pp. 1-5.

[6] S. Duman, U. Güven, Y. Sonmez, and N. Yrükeren, "Optimal power flow using gravitational search algorithm," in Energy Conversion \& Management, vol. 59, pp. 86-95, Jul. 2012.

[7] M. R. Adaryani and A. Karami, "Artificial bee colony algorithm for solving multi objective optimal power flow problem," in International Journal of Electrical Power \& Energy Systems, vol. 53, no. 1, pp. 219$230,2013$.

[8] B. K. Panigrahi, P. N. Suganthan, S. Das, and S. C. Satapathy, "Optimal power flow for Indian 75 bus system using differential evolution," in [Lecture Notes in Computer Science] Swarm, Evolutionary, and Memetic Computing, vol. 7076, pp. 110-118, 2011.

[9] M. U. Keerio, A. Ali, M. Saleem, N. Hussain, and R. Hussain, "Multiobjective optimal reactive power dispatch considering probabilistic load demand along with wind and solar power integration," in Proceedings of 2020 2nd International Conference on Smart Power \& Internet Energy Systems (SPIES), Bangkok, Thailand, 2020, pp. 502-507.

[10] M. T. Al-Hajri and M. A. Abido, "Multiobjective optimal power flow using improved Strength Pareto Evolutionary Algorithm (SPEA2)," in 2011 11th International Conference on Intelligent Systems Design and Applications, 2011, pp. 1097-1103.

[11] I. Sharma, K. Bhattacharya, and C. Cañizares, "Smart distribution system operations with price-responsive and controllable loads," in IEEE Transactions on Smart Grid, vol. 6, no. 2, pp. 795-807, Mar. 2015.

[12] J. Hou, Y. Xu, J. Liu, L. Xin, and W. Wei, "A multi-objective volt-var control strategy for distribution networks with high PV penetration," in Proceedings of 10th International Conference on Advances in Power System Control, Operation \& Management (APSCOM 2015), Hong Kong, China, 2015, pp. 1-6.

[13] S. S. Reddy and B. K. Panigrahi, " Optimal power flow using clustered adaptive teaching learning-based optimization," in International Journal of Bio-Inspired Computation, vol. 9, no. 4, pp. 226-234, 2017.

[14] S. S. Reddy, "Multi-objective optimal power flow for a thermal-wind- 
solar power system, " in Journal of Green Engineering, vol. 7, no. 4, pp. 451-476, 2018.

[15] Y. Li, R. Yang, and X. Zhao, "Integrated reactive power optimization method for active distribution networks based on a Quantum Krill Herd Algorithm," in Electric Power Components and Systems, vol. 47, no. 2, pp. 1-15, Dec. 2019.

[16] J. Cheng, Y. He, H. Cao, H. Shi, J. Hu, and T. Ding, "Optimal configuration of distributed generation for improving voltage quality based on adaptive weighted PSO," in Proceedings of 2020 19th International Conference on Harmonics and Quality of Power (ICHQP), Dubai, United Arab Emirates, 2020, pp. 1-6.

[17] H. Wang, H. Jiang, K. Xu, and G. Li, "Reactive power optimization of power system based on improved particle swarm optimization," in Proceedings of 2011 4th International Conference on Electric Utility Deregulation and Restructuring and Power Technologies (DRPT), Weihai, China, 2011, pp. 606-609.

[18] Y. Ai and J. Ding, "Study about optimization scheduling method of economic benefit for grid-connected household photovoltaic systems," in IEEJ Transactions on Electrical and Electronic Engineering, vol. 15, no. 4, pp. 527-535, Apr. 2020.

[19] J. Lamb and B. Mirafzal, "Active and reactive power operational region for grid-tied inverters," in Proceedings of IEEE International Symposium on Power Electronics for Distributed Generation Systems, pp. 1-6, Jun. 2016.

[20] K. Deb and H. Jain, "An evolutionary many objective optimization algorithm using reference-point-based non dominated sorting approach Part I: Solving problems with box constraints," in IEEE Transactions on Evolutionary Computation, vol. 18, no. 4, pp. 577-601, Aug. 2014.

[21] K. Deb, L. Thiele, M. Laumanns, and E. Zitzler, "Scalable multiobjective optimization test problems," in Proceedings of the 2002 Congress on Evolutionary Computation. CEC'02 (Cat. No.02TH8600), Honolulu, HI, USA, 2002, pp. 825-830, vol. 1.

[22] L. R. Araujo, D. R. R. Penido, S. Carneiro, and J. L. R. Pereira, "A threephase optimal power-flow algorithm to mitigate voltage unbalance," in IEEE Transactions on Power Delivery, vol. 28, no. 4, pp. 2394-2402, Oct. 2013.

[23] W. H. Kersting, in Distribution System Modeling and Analysis, CRC Press, 2002.

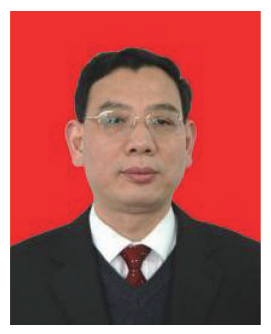

Yongle Ai was born in China. He received the B.S. degree in power electronics from Xi'an University of Technology, Xi'an, China, in 1987, the M.S. degree in electrical engineering from Southwest Jiaotong University, Chengdu, China, in 1995, and the Ph.D. degree from the University of Stellenbosch, Stellenbosch, South Africa, in 2006.

He joined the Henan Polytechnic University from 1987 to now. He is currently a Professor at the School of Electrical Engineering and Automation, Henan Polytechnic University, China. His research interests include multiphase AC machine control and power system filter. Prof. Ai is a member of the Chinese Society for Electrical Engineering.

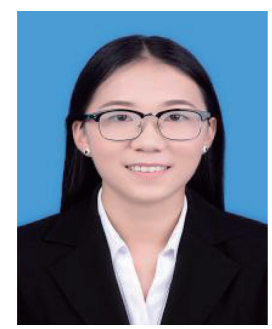

Mingzhu Du was born in China. She received the B.S. degree in electrical engineering and automation from Zhengzhou University of Aeronautics, Zhengzhou, China, in 2018. She is currently pursuing a M.S. degree in electrical engineering and automation at Henan Polytechnic University. Her current research interests include new technology of electrical energy and system optimization analysis.

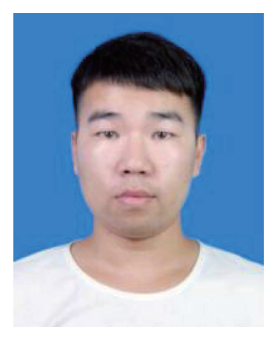

Zhihang Pan was born in China. He received the B.S. degree in electrical engineering and automation from Henan University of Engineering, Zhengzhou, China, in 2019. He is currently pursuing a M.S. degree in electrical engineering and automation at Henan Polytechnic University. His current research interests include new technology of electrical energy and microgrid control.

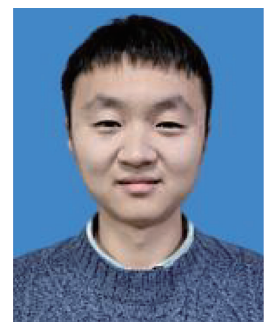

Gangxing Li was born in China. He received the B.S. degree in electrical engineering and automation from Henan Normal University, Xinxiang, China, in 2019. He is currently pursuing a M.S. degree in electrical engineering and automation at Henan Polytechnic University. His current research interests include new technology of electrical energy and microgrid control. 